\title{
Envisioning the future of cultural neuroscience
}

\author{
Shinobu Kitayama and Steve Tompson \\ Department of Psychology, University of Michigan, Ann Arbor, Michigan, USA
}

In the present commentary, we first examine the three target articles included in the Asian Journal of Social Psychology special issue on cultural neuroscience. We spell out the contributions that the articles have offered to the field. We extend this examination with our own theoretical model of neuro-culture interaction, which proposes that brain connectivity changes as a function of each person's active, repeated engagement in culture's scripted behavioural patterns (i.e. practices). We then locate the current endeavour of cultural neuroscience within a broader framework, detailing empirical, theoretical, and meta-theoretical reasons why the approach of cultural neuroscience is important to both socio-behavioural and biological sciences. It is concluded that the scholarship demonstrated in the target articles will be an important collective asset for all of us who aspire to understand the human mind as fundamentally biocultural and to study it as such.

Key words: brain, cultural neuroscience, culture, epigenesis, neuroplasticity.

\section{Introduction}

How will culture influence the brain and, conversely, how will the brain be part of a sociocultural dynamic that constitutes the daily social reality? Is the field ready to tackle these big questions that encompass what appears to be the most micro of micro-processes in social life (the brain) and what would seem to be the most macro of macro-processes (culture)? Or, if not, is it really wise to even give it a shot? One can further ask if there is anything new in the allegedly new field of cultural neuroscience. Isn't it just old wine in a new, fashionable bottle - just yet another fad that will go away sooner or later? Most of all, one could object to the field on the ground that cultural neuroscience is intolerably reductionistic: How can dynamic sociocultural processes be explained by neurons?

These are some of the questions sceptics might immediately ask on the field of cultural neuroscience that has just begun to emerge over the last few years (Chiao \& Ambady, 2007; Han \& Northoff, 2008; Kitayama \& Park, in press; Kitayama \& Uskul, 2010). These questions would seem reasonable and, at least to some extent, they are well justified. If, for example, cultural neuroscience should claim to explain the sociocultural solely in terms of the brain, the field would surely be guilty of reductionism.

In view of these and probably numerous other questions on cultural neuroscience, the three target articles included in the Asian Journal of Social Psychology's special issue on cultural neuroscience are especially welcome. They can serve both as introductory reviews to the field and as an

Correspondence: Shinobu Kitayama, Department of Psychology, University of Michigan, Ann Arbor, MI 48109, USA. Email: kitayama@umich.edu

Received 11 April 2010; Accepted 16 April 2010. excellent source for research ideas. And, most of all, they offer much needed reassurance that there is something to 'it', thereby inviting many promising young scholars both in Asia and elsewhere to the field. In fact, these articles make it very clear that the sceptics would at least have to carefully examine what the field has offered so far and to listen to what its advocates have argued. Once the future of this field is seen from its advocates' point of view, very different scenery may begin to emerge - this scenery is that of a frontier that is just waiting to be discovered and exploited to yield a major advancement in the sciences of both culture and brain.

The three articles are thus quite timely. Each of them is a concise and well-written treatise that must be read carefully by all students of this new field. Furthermore, the scholarship demonstrated here is first rate. The thesis in each case is thought-provoking and intellectually highly stimulating. The three articles, with eight cutting-edge scholars together as a group, have taken stock of previous theories and findings in both cultural and cross-cultural psychology and cognitive and social neuroscience. They then have gone a big step further by envisioning the future of the project of both using neuroscience as yet another set of tools to investigate cultural processes and, conversely, using culture as a context in which to further examine neuropsychological processes of the human mind.

\section{Main points revisited}

\section{Zhou and Cacioppo}

The piece by Zhou and Cacioppo (2010) is perhaps the most general and encompassing of the three target articles, insofar as their goal is to situate the emerging field of cultural neuroscience within a multi-level framework that 
connects the biological to the social and vice versa. The authors argue that current behavioural paradigms of cultural and social psychology need to be integrated with methods and approaches of neuroscience. They maintain that examining the mutual relationship between sociocultural and neurobiological processes is essential to understanding the most fundamental aspects of human nature. In supporting this argument, Zhou and Cacioppo highlight how functional magnetic resonance imaging (MRI) methodology can be integrated with various cultural psychological approaches to yield fruitful and potentially groundbreaking research. They emphasize that social neuroscience techniques have the ability to differentiate between underlying neural processes and address cultural differences in mental processes. Of importance, some of the mental processes that can be studied with functional MRI (fMRI) and other neuroscience measures might not necessarily result in any behavioural differences and, thus, could be beyond scientific investigation without the current methods of neuroscience.

Zhou and Cacioppo (2010) finish with a list of issues which future researchers should be wary of when using fMRI to examine culture and the brain. While many of these caveats simply highlight the current limitations of fMRI technology which apply to any fMRI researcher, some are of special significance for cultural neuroscience. We will return to some of these issues later in the present paper.

\section{Ames and Fiske}

Ames and Fiske (2010) take a somewhat different approach. Their aim is to provide a panoramic view of the current field of cultural neuroscience. They start out by noting how counter-intuitive it may initially appear that the combination of two seemingly incompatible disciplines cultural psychology and neuroscience - can result in significant mutual benefit to both. They provide a compelling argument for not only the necessity of combining the two approaches, but also for the need to look across multiple domains to examine the biological underpinnings of culture. As they note, the human brain is 'biologically prepared to acquire culture: The ability to coordinate thoughts and behaviours within social groups has aided primate and hominid survival' (p. 72).

Ames and Fiske (2010) have done an admirable job to bring together, and provide evidence for, cultural differences in brain functioning across a diverse range of psychological phenomena. Their review extends from basic cognitive processes (e.g. object processing, colour and taste perception) to complex social psychological processes (e.g. mental representations of the self and others, trait/belief inference). For example, they discuss a study by Gutchess, Welsh, Boduroglu, and Park (2006) which suggests that cultural differences in object processing are related to increases in activation in areas of the brain associated with object processing among Westerners, rather than increases in activation in areas of the brain associated with background processing among Easterners. Ames and Fiske present this as an example of how cultural neuroscience could empirically test two competing hypotheses that suggest the same behavioural predictions. They conclude with suggestions for areas that may yield fruitful cultural neuroscience research in the near future.

\section{Ng, Han, Mao, and Lai}

Unlike the first two articles, the third and final article from this AJSP special issue is an empirical study. Obviously narrower in scope than either of the earlier two, the article by Ng, Han, Mao, and Lai (2010) nonetheless offers something that is just as fundamental in its own way. This paper is a prime example demonstrating many of the ideas discussed in the two other papers in this issue. Specifically, they present empirical work which demonstrates how a sociocultural framework can be used with fMRI to show real cultural differences in neural activity. They conceptually replicate the findings of Zhu, Zhang, Fan, and Han (2007), showing that whereas neural representations of the self and others (even significant others) are differentiated in the Western brain, the neural structure of the self in Chinese is more closely connected with others, as indicated by activity in the ventral medial prefrontal cortex (VMPFC).

Importantly, $\mathrm{Ng}$ et al. (2010) extend the Zhu et al. (2007) findings in two significant ways. First, they show that the strong overlap between self and significant others extends beyond significant others with whom one identifies (i.e. mother), to others who play an important role in one's life, but with whom they do not identify (non-identified person or NIP). More significantly, they use a within-subjects design, including only bicultural participants from Hong Kong who rate high in both Chinese and Western selfidentity, which rules out confounding variables such as language or genetics, and shows that people with strong bicultural identities have both independent and interdependent neural representations of the self, which can be differentially activated depending on the cultural context.

\section{Cultural neuroscience as an integrated approach to human psychology}

Admirable and extremely useful in their own ways, however, the three articles left out some issues that must be brought back to the foreground and fully discussed for their implications. In our opinion, none of the three papers offers an adequate conceptualization of what culture is. Culture is a multifaceted concept, which encompasses attitudes, beliefs, and other social cognitions, as well as social repre- 
sentations and socially shared ideas. Moreover, daily scripted routines called practices or cultural tasks, as well as social institutions and other systems of social interaction, are also of importance (Kitayama, Conway, Pietromonaco, \& Park, 2010). It is not at all clear which aspects of culture may be significant in cultural neuroscience and why.

Another issue stems from the fact that developmental perspectives are missing in all three articles. It is surely interesting to show that cultures are different. It is even more interesting to show that this difference can be observed in brain responses. Yet, if one is to hope to understand the nature of cultural influence, it is inevitable, at some point, to start asking much more difficult questions of why and how such differences have come about over time. These questions must be formulated at the levels of evolution, history, and individual development. We are well aware that some may argue that it is a bit too much and, thus, a bit unfair to raise these 'big questions'. Yet, even though the field is not quite ready to tackle them, it is well advised to start thinking about and analyzing them. This theoretical effort may have some positive ramifications even though such big questions might prove to be too difficult to handle at the present moment.

What we wish to add to the already impressive collection of papers is to address these questions. We will start by providing a general theoretical framework of our own in which to locate major points made by each target article and then draw some additional implications from it. We will then discuss some new directions for the new field of cultural neuroscience.

\section{Neuro-culture interaction model}

Our theoretical framework, called the neuro-culture interaction model (Kitayama \& Park, 2010; Kitayama \& Uskul, 2010), is presented in Figure 1. The model draws on a time-honoured idea that culture constitutes an external environment by providing a number of scripted behavioural routines called practices (see e.g. Shweder, 1990). For example, contemporary American society offers a number of practices, such as show-and-tell and publish-or-perish, that are tied closely to values of independence such as self-expression and personal achievement. The practices are historically produced as a function of a variety of macro-level factors such as ecology, subsistence systems, population density, residential mobility, social and political systems and institutions (Box 1 of Fig. 1). They are further weaved into collectively shared ideologies such as individualism, collectivism, honour, and nationalism, among others. Scripted behavioural routines or practices which a cultural group has accumulated across generations constitute the reservoir of the culture's wisdom and practical knowledge about how to survive, what is good and beautiful, and how good and beautiful things can be achieved or attained (Box 2 of Fig. 1).

One key idea underlying this model is that cultural wisdom as represented in the pool of practices is acquired and accumulated in the brain as new members of the culture begin engaging in some of the practices, insofar as brain connectivity is likely to change as a function of active, wilful engagement in scripted behavioural routines (Boxes 3 and 4 of Fig. 1). The perspective of the neuro-culture interaction model is thus fundamentally developmental.

For example, Draganski et al. (2004) found that training in juggling leads to structural changes in brain areas associated with the processing and storage of complex visual motion. This study showed that repeated engagement in a task over a relatively short period of time (3 months in this study) can lead, not only to functional changes in cortical activity, but also to anatomical changes in cortical structure. To the extent that the task is therefore more or less prominent in a given culture, we may expect that repeated engagement in the task should lead to cultural differences in functional connectivity and, perhaps, in the physical structure of the brain as well. Now, basically the same point has been repeatedly made in respect to the abacas (Hanakawa, Honda, Okada, Fukuyama, \& Shibasaki, 2003), musical instruments (Muente, Nager, Beiss, Schroeder, \& Altenmueller, 2003), and cab driving

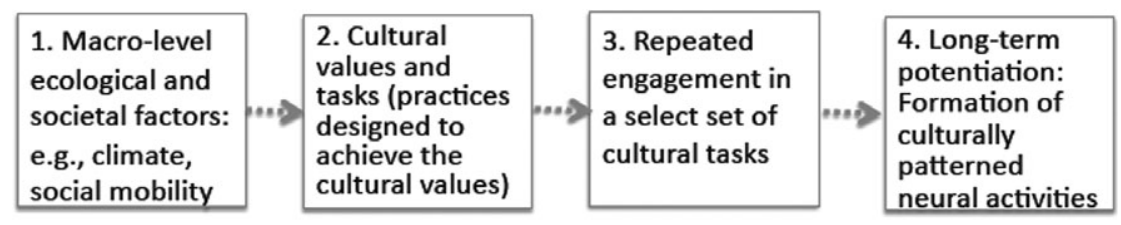

\begin{tabular}{|c|c|c|}
\hline \begin{tabular}{|l|}
$\begin{array}{l}\text { 5. Spontaneous } \\
\text { enactment of } \\
\text { culturally } \\
\text { scripted } \\
\text { behaviours when } \\
\text { such behaviours } \\
\text { are called for. }\end{array}$ \\
\end{tabular} & \begin{tabular}{|l|l|} 
& 6. Establishing \\
one's identity
\end{tabular} & \begin{tabular}{|l|l} 
7. Biological \\
adaptation as \\
assessed by \\
reproductive \\
success
\end{tabular} \\
\hline
\end{tabular}

Figure 1 Neuro-culture interaction model. Adopted from Kitayama and Uskul (2010) with modifications. 
(Maguire et al., 2000) among others. Very much likewise, once people engage in their own culture and start acting in accordance with the culture's scripted patterns for action, their brain changes accordingly.

One important point to keep in mind is that if engagement in cultural practices is to change a person's brain, the engagement may have to be wilful, voluntary, and active. When individuals conduct a certain behaviour willingly and voluntarily, they will represent their own goal as something they really want to achieve, attend to a variety of relevant stimuli in the environment with curiosity, seek out relevant stimuli vis-à-vis the personal goal while avoiding or ignoring some others, and coordinate their behaviour accordingly. Most importantly, achieving the personal goal is likely to produce an internal reward - a positive hedonic state - that reinforces the entire sequence of curiosity, attention, and action coordination. In contrast, when they regulate their behaviour vis-à-vis an externally imposed normative goal, they may not show as much curiosity in the environment. Moreover, they may do what they have to do while referring to some obviously relevant environmental stimuli with minimum effort. Most of all, internal rewards are much less likely to be involved. It is our hypothesis, then, that brain circuitry is more likely to be stimulated, new neurons generated and unnecessary ones pruned when the behaviour is regulated in reference to one's personal goal and thus is a source of internal reward, but that the plastic brain change is increasingly less likely when the behaviour is situationally controlled.

This point has been clearly demonstrated in animal studies, which indicate that repeated actions can have substantial impacts on the production of new neurons and subsequent modification of brain connectivity only if the actions are voluntary. van Praag, Kempermann, and Gage (1999) found that voluntary engagement in physical activity resulted in significant increases in the production of neurons in the mouse hippocampus. Importantly, forced engagement in physical activity did not result in any increases in hippocampal neurogenesis.

It is reasonable to assume that individuals are likely to engage in their culture in an active, voluntary, and persistent fashion if they identify with the culture and their selfconcept and values are congruous with the values sanctioned by their culture. For example, Western cultures place a strong emphasis on independent or individualistic values such as self-promotion, self-uniqueness, and personal achievement. Yet, individuals may vary considerably in the degree to which they endorse these foundational values of culture (Schwartz, 1992). The more committed they are to these values the more likely it would be for them to actively, repeatedly, and voluntarily engage in the culture's practices, with correspondingly larger consequent changes in their brain connectivity.
Once changed through engagement in cultural practices, the brain may be expected to influence subsequent behaviours, both covert (e.g. attention and reasoning) and overt (e.g. social behaviour). However, any behaviour is multiply determined and regulated and the culturally modified brain connectivity is only one of many factors that can influence any given behaviour. Among others, if any given behaviour is inconsistent with situational norms, it will be interrupted or inhibited. It is likely, then, that the culturally modified brain connectivity will foster the relevant culturally scripted behaviour only when the behaviour is normatively sanctioned and thus strongly called for in a given social situation (Box 5 of Fig. 1).

Although this point might seem obvious given a number of studies in social psychology demonstrating the power of social situation in influencing social behaviour (Ross \& Nisbett, 1991), its implications can be far-reaching. In particular, Kitayama and Uskul (2010) have argued that spontaneous and seamless behaviours that are normatively sanctioned culturally and strongly called for by a given situation can affirm the person as a good cultural member in the eyes of both the self and others who witness the behaviours (Box 6 of Fig. 1). The recognition of the self as a good cultural member will create and reinforce the person's cultural identity, consolidating his or her commitment to the values of the culture. At the same time, the comparable recognition by others will enhance the self's reputations in the cultural community as a good and committed cultural member, thereby improving and securing the stature of the self within the community. And, in the long run, this will have other positive consequences, increasing the likelihood of the person succeeding socially, economically and, eventually, reproductively (Kitayama \& Park, 2010; Kitayama \& Uskul, 2010) (Box 7 of Fig. 1).

\section{Some implications of the neuro-culture interaction model}

The neuro-culture interaction model can be used as a heuristic in making some suggestions on possible forms of culture-brain interaction. We believe that some of the insights suggested by the three target articles are quite congruous with such implications of the neuro-culture interaction model. We found three noteworthy points.

Connectivity is crucial. First, the neuro-culture interaction model suggests that brain connectivity should change as a result of persistent and willing engagement in some specific set of cultural practices. It is connectivity, rather than the absolute level of activation of any given brain regions per se, that is influenced by culture. Quite consistent with this analysis, Zhou and Cacioppo (2010) discuss the importance of examining the connectivity between different brain regions, rather than examining the brain regions in isola- 
tion. While an analysis regarding connectivity certainly leads to predictions that cultural differences would exist in the relative strength of neural activity among neurons within a given brain region, it also would predict that cultural differences should exist in the relative strength of activity among neurons in different brain regions. Therefore, single region analyses ignore a large set of potential cultural differences. While the research discussed in this issue primarily focuses on activity within regions of the brain, the overwhelming evidence for within-region cultural differences would lead one to predict that there should also be a bounty of cultural differences in the connectivity between brain regions.

Predicting brain responses with self-belief measures of values, self, and identity. Following the logic of the neuroculture interaction model, one might suggest that it is not culture in the sense of group membership per se, but rather culture in the sense of one's subjective commitment to the culture's foundational values and associated practices that produce relatively enduring brain changes. This means, for example, that if Sam is committed to his own cultural group's foundational values of independence, he is likely to actively, willingly, and repeatedly participate in the culture's practices that are linked to independence. This should, in turn, leave relatively enduring brain traces that produce a variety of cognitive, emotional, and motivational biases that are congruous with the relevant practices. One striking implication of this analysis is that brain responses are likely to be predicted by each person's values, selfconcepts, and other attitudes that predispose the person to engage in the pertinent cultural practices. In fact, one finding that is hidden behind some of the studies reviewed by Ames and Fiske (2010) is that brain measures indicative of independence (or interdependence) are often reliably predicted by self-reported levels of independent (or interdependent) social orientation as assessed by conventional questionnaire scales (Kitayama \& Uskul, 2010). This emerging evidence might not surprise anyone, except those, like us, who have conducted research in the area of culture over years. The fact is that the corresponding correlations are typically very hard to observe with behavioural measures as a dependent variable (e.g. Kitayama, Park, Servincer, Karasawa, \& Uskul, 2009).

The finding that self-belief measures of cultural values are far more likely to predict neural, rather than behavioural, responses may seem quite puzzling at first glance because it is unlikely to be an artifact of either low reliability of the behavioural measures (because the test-retest reliability of these measures are often reasonably high) or the inflated likelihood of Type-I errors that is allegedly common in imaging research (Vul, Harris, Winkielman, \& Pashler, 2009, because this arguably is not the case with specific event-related potential [ERP] measures) (see
Kitayama \& Uskul, 2010). The finding, however, may begin to make sense once some aspects of the neuro-culture interaction model are taken seriously. The model predicts that neuroplasticity should only arise from willing and repeated engagement in a particular culture's practices. Accordingly, level of cultural identity should be an important factor in predicting this engagement. Hence, high cultural self-identity should lead to greater willingness to voluntarily and continuously participate in a culture, and result in the subsequent, long-term changes in neural connections. However, engagement in practices of a culture one does not identify with may lead to certain behavioural tendencies that are instigated by situational norms, but may not lead to any long-term changes in neural activity because, in such cases, the engagement is more likely to be seen as forced or driven by external factors. These behaviours may then not be related either to the self-identity or to culturally shaped brain connectivity.

Significance of systematic participant sampling. While providing important information on culture-brain interaction, the systematic individual difference predicted by the degree of engagement in culture raises a serious concern in sampling. This is especially true, as Zhou and Cacioppo (2010) pointed out, because the standard for sample size in neuroscience research is significantly smaller than what is typically used in cultural psychology research. With smaller sample size comes larger sampling error and, as a result, there is a greater risk that the sample is not representative of the culture being studied. Additionally, as most neuroscience research has used Western populations, and neuroscience research is relatively newer to Eastern countries, there could be some sampling biases related to perceptions of risk involved in neuroscience research, especially in studies that use methodology that nonWestern populations may be less familiar with, such as fMRI.

One useful measure of precaution might be to carry out a large-scale survey study first and measure potential participants' values, self-concepts, and identities and use this information in participant recruitment. For example, participants may be recruited equally from the entire range of distribution of, say, independence or interdependence. Alternatively, they may be recruited from each of the two extremes of the distribution. In either case, this systematic sampling method pre-empts biased sampling that can seriously distort the data especially when the $N$ is quite small.

In this regard, we found it quite commendable that $\mathrm{Ng}$ et al. (2010) used an explicit criterion to select their experimental participants. Previous research suggests that for participants low in cultural identity or whose bicultural identities are in conflict, cultural cues may elicit patterns of behaviour opposite to those espoused by the culture being cued (Mok \& Morris, 2009). Therefore, it is quite possible 
that participants low in cultural identity would not show the same results as those in the $\mathrm{Ng}$ et al. study when primed with a culture they had low self-identity for. Thus, their results may specifically hinge on their subject recruitment and, without their systematic subject selection, these results may not be interpretable.

\section{Three reasons why we need cultural neuroscience}

So far, we have reviewed the three target articles and in doing so we have also introduced our own theoretical model for the culture-brain interaction. It is clear that our own thinking resonates well with the three target articles in several key points. Furthermore, both the theory and the empirical data of cultural neuroscience now seem strong enough to counter many of the criticisms and questions sceptics might level against this new endeavour.

Here, then, we would like to go back to the questions we attributed to sceptics at the beginning of this commentary. We noted that the questions are real and, to some extent, very well justified. In fact, under some different guises, they have often been heard argued by researchers of some neighbouring disciplines. After having examined the three target articles in some detail and presented our own theoretical approach, we wish to address these questions head on. Fortified with ideas and data taken stock so far in this commentary, this effort may enable us to assess the present state of cultural neuroscience from an overarching perspective and then to envision future directions for the field.

As shall be made clear, despite all the criticisms that can be levelled against cultural neuroscience, we believe that cultural neuroscience has substantive potential to make important contributions to both social and behavioural sciences and biological sciences. There are three important reasons for this assessment. These reasons focus, respectively, on empirical, theoretical, and meta-theoretical considerations for cultural neuroscience.

\section{Empirical reason: Neuroscience measures provide information that is unique and indispensable for theory building}

We started out our own cultural neuroscience project just a few years ago. Back then, our primary motivation was rather simple and, one could say, rather naïve: We just wanted to see how deep culture might go 'under the skin'. To address this question, it is obviously important to use measures that tap processes within the skull in more or less direct fashion. From this fact alone, neuroscience measures such as fMRI and ERP can provide important information about how culture might get into the brain in ways that are simply impossible to gauge with behavioural measures (e.g. self-report, judgment, memory, response time, performance) alone.

As shown in a review by Ames and Fiske (2010), the progress of empirical work in cultural neuroscience in the last few years is remarkable. It is now very clear that our original question has received an answer and, yes, culture does indeed go under the skin. More importantly, however, the progress that occurred in the interim has shown abundantly the ability of the neuroscience measures to address important theoretical questions as well.

To illustrate, consider the theory of cognitive dissonance - arguably the most important theory in social psychology during the last century. The original theory claimed that attitude-inconsistent behaviour causes a negative arousal, called dissonance (Festinger, 1957). Because dissonance is aversive, people are motivated to reduce it by revising their attitude to restore the state of consonance. Researchers have worked very hard to demonstrate the actuality of the negative arousal as a guiding force of the observed attitude change (Cooper, Zanna, \& Taves, 1978). As it turned out, this effort was very difficult, if not utterly impossible, with behavioural measures alone. Neuroscience measures would enable us to fill the gap. We now know that certain brain areas that are linked to conflict monitoring (ACC) and negative somatic arousal (anterior insula) are strongly activated when people behave in an attitude-incongruous fashion and, moreover, these activations do predict subsequent attitude change (van Veen, Krug, Schooler, \& Carter, 2009). At least among Caucasian Americans, the negatively arousing state called dissonance is real and functionally linked to attitude change. This finding must be expanded cross-culturally insofar as the conditions in which dissonance occurs are likely to vary considerably across cultures depending on the nature of the self that is sanctioned in a given culture (Kitayama, Snibbe, Markus, \& Suzuki, 2004).

In addition to their ability to enable us to have direct access to underlying processes (e.g. dissonance in the above example), neuroscience measures can be useful in some other ways as well. In particular, online measurement of electrical activity on the scalp as participants carry out certain cognitive tasks (called ERP) enables us to observe processes more directly as they occur over time. We now know, for example, that when people hear someone speak a word in a vocal tone that is inconsistent with the meaning of the word, the inconsistency is detected quite rapidly, as early as 300-400 ms post stimulus (Ishii, Reyes, \& Kitayama, 2003). Evidence, in fact, is very strong that the ability to detect inconsistent word meaning vis-à-vis its background vocal tone is substantially higher for females than for males when assessed with the ERP measure (Ishii, Kobayashi, \& Kitayama, 2010; Schirmer \& Kotz, 2003). Surprisingly, however, the gender difference rarely shows up when the seemingly identical phenomenon is tested with 
behavioural measures such as response time and judgment accuracy (Ishii et al., 2003). In this case, it is quite clear that the brain measure is tapping processes that are totally missed by the behavioural measures. Some other similar examples are discussed in the Zhou and Cacioppo (2010) article.

Remember our earlier discussion on the ability of selfbelief measures of, say, independence and interdependence to predict brain measures of independence or interdependence, but not their behavioural counterparts. Kitayama and Uskul (2010) used this observation as evidence for an aspect of the neuro-culture interaction model. That is, the model proposes that self-beliefs predict how persistently and earnestly one engages in pertinent cultural practices, which, in turn, predict corresponding changes in brain connectivity. However, behavioural habits can be influenced by numerous other factors including, among others, situational norms. Aside from the eventual validity of this theoretical analysis, it is quite obvious that the neuroscience measures are again tapping processes (i.e. accumulation and representation of cultural information in the brain) that the traditional behavioural measures find hard to handle.

\section{Theoretical reason: Culture and the brain make each other up}

Ames and Fiske (2010) started out their target article by pointing out the seemingly contradictory nature of the effort to combine culture with the brain. Through their meticulous review of emerging cultural neuroscience evidence, the authors successfully showed that this effort is necessary and potentially successful. We are in full agreement with them.

For some time, it has been argued that the mammalian brain, in general, and the human brain, in particular, are fundamentally social (Dunbar, 2007) - a point echoed by Zhou and Cacioppo (2010). In fact, the human brain by design needs a massive amount of external input for it to be fully functional (Stiles, 2008). It goes without saying, then, that the brain requires culture. With increasing research effort on neuroplasticity in recent years, this point will, in all likelihood, be demonstrated even more powerfully in the near future. Simple as it may be, the point deserves emphasis as it illustrates the urgent need for the mainstream neuroscience research to incorporate culture into its purview.

Conversely, cultural psychology needs neuroscience as well, not just because neuroscience measures provide nonredundant information (as shown above), but also, more crucially, because the brain is an indispensable element for culture to work. Within the framework presented by the neuro-culture interaction model, culture is composed of scripted routines called cultural practices. These practices are associated with a variety of ideas and beliefs. And most importantly, these practices represent a crucial medium by which the brain is modified. People who are committed to the values and worldviews of the culture are likely to carry out the culture's practices persistently and earnestly, with resulting changes in brain connectivity. Culture is enlivened and, thus, can become a powerful determinant of human mentalities because of its ability to engage the brain as its part. In short, it is not too far-fetched to say, following Rick Shweder's (1990) proclamation two decades ago that culture and the brain, in a very literal sense, make each other up.

If framed as a constituent element of culture, the brain can no longer be seen as a sole engine that causes unidirectional influences on all other processes. Whereas the brain is a site that accumulates and embodies information stemming from dynamic sociocultural patterns, culture is a rich source of symbolic and behavioural resources that make it possible for the brain to grow and become fully functional. Far from reductionistic, cultural neuroscience, if properly understood and practiced, would be the study of a comprehensive system of human biological adaptation that encompasses both collective-level processes of ecosocial systems (including 'culture' narrowly defined) and neuro-physiological processes of the brain.

\section{Meta-theoretical reason: Integrating nature and nurture}

The first two reasons we cited pertain to both empirical considerations (empirical reason) and theoretical considerations (theoretical reason) that suggest the proposed integration between cultural psychology and neuroscience will be highly fruitful and productive. These considerations, however, may be expanded and reinforced with some additional considerations that are more meta-theoretical. They pertain to human evolution and genetic and epigenetic processes that are likely to be closely tied to both brain and culture. We call these considerations meta-theoretical only because little or no published research exists today that investigates these issues up-front. This will change soon in all likelihood (see Chiao \& Blizinsky, 2010, for a pioneering effort). When that happens, cultural neuroscience will have bridged natural sciences and social sciences, thereby overcoming the unfortunate division of labour between the two types of sciences.

One important event that is likely to have precipitating impacts has already taken place. The National Institute of Mental Health sponsored a highly cross-disciplinary by-invitation-only conference on culture, evolution, and the brain at the Center for Advanced Study in the Behavioral Sciences (CASBS) in Stanford, California, on 26-27 March, 2010. One of us (Kitayama) co-organized the conference with a developmental psychologist (Anne Petersen) and a primatologist (Stephen Suomi). The three of us literally witnessed the collaborative spirit and the intellectual 
excitement that permeated the entire conference. In our assessment, all participants including ourselves strongly sensed a new field in its making.

So far, cultural research, both behavioural and neural, has focused on cross-cultural variations in behaviour and in the brain. The horizon of this endeavour will be immediately expanded once it is realized that human evolution did not stop when culture was born. Hawks and colleagues (e.g. Hawks, Wang, Cochran, Harpending, \& Moyzis, 2007) have presented a strong case for an exponentially increasing rate of active genetic selections over the last 10000 years. These selections can be very minor, and are likely to account for only a miniscule amount of genetic variation in humans today. In fact, they are largely confined to what may be called single nucleotide polymorphisms (SNPs). Yet, given appropriate external conditions, a small change can cause large effects. Thus, consequences of a miniscule genetic change on body morphologies and psychological functions can sometimes be quite sizable and crucially important in understanding local forms of adaptation.

The active genetic selections over the last 10000 years are made possible by both increasing population size and high population density, both fostered and reinforced by the invention of agriculture. SNPs are thus likely to be highly local and contingent on specific modes of ecological, sociocultural, and even linguistic adaptations (Cochran \& Harpending, 2009). Until just a few years ago, it was quite reasonable to postulate that culture is a matter of learning and biology is a matter of organismic design. This division of labour is no longer possible and, in fact, it is likely to impede progress of the field if rigidly endorsed. Quite literally, culture can provide a dynamic context for genetic selection, while, at the same time, genes have the potential to be an extremely powerful force that motivates certain forms of culture in lieu of others.

Most excitingly, the CASBS conference highlighted the fact that recent advancement in epigenetic processes has provided, in broad outline, how one might begin to combine the two disparate fields of research, namely, cultural variation in mentality and brain on the one hand and highly local and relatively recent (less than 10000 years old) genetic selections on the other hand. Recent epigenetic research has demonstrated that gene expressions are contingent on daily experiences throughout the life span. At least in some cases, detailed mechanisms have been worked out through which external environmental inputs (e.g. trauma early in life) can change the expression of certain genes, resulting in considerable behavioural differences (e.g. depression, aggressiveness, or violence) later in life (Ian, Weaver, Meaney, \& Moshe, 2006). Given this new development, it is now conceivable to envision certain mechanisms by which recurrent behaviours that are patterned by culture can plausibly trigger a cascade of neuro- physiological events that eventually cause significant differences in the expression of certain key genes, which could, in turn, play important roles in forming relevant brain-processing pathways. The resulting neural complex of the brain predisposes the person to act in certain ways, which, in turn, influences his or her reproductive success, thereby eventually contributing to the population-level prevalence of the genes involved.

As in all cases in science, the devil is in the details. Admittedly, the details have yet to be investigated, let alone specified. Nevertheless, the foregoing theoretical blueprint that integrates culture, genes, and the brain was simply not available until just a few years ago. At a minimum, the emerging theoretical blueprint will likely influence what we study and how we study it, thereby having significant long-term effects on how the field unfolds in the next decade. We are, therefore, hopeful that the future effort to expand the current neuroscience in this new direction can have far-reaching consequences on our very basic understanding about human nature. Once achieved, this new understanding will have overcome the current dichotomy between the sociocultural and the biological, thereby presenting a truly integrated image of the human as both biological and cultural. We thus submit that the sociocultural can provide a very exciting new frontier for neuroscience. In all likelihood, by exploiting this new frontier, our understanding of the sociocultural will also have been radically transformed.

\section{Conclusions}

We started out with a critical examination of the three target articles included in the AJSP special issue on cultural neuroscience. We spelled out the contributions that the articles have offered to the field. We extended this examination with our own theoretical model of culture-brain interaction. We then sought to locate the current endeavour of cultural neuroscience within a broader framework, detailing three reasons why the approach of cultural neuroscience is important to both social and biological sciences.

In our judgment, this commentary will have succeeded if we can convey our own excitement about the effort to integrate cultural psychology and neuroscience and, more importantly, our own sense of mission - a mission to see to it that cultural neuroscience fulfils its promise. As in all such efforts, envisioning the future is one thing, and creating it is quite another. Yet, the vision is essential in influencing what we can eventually craft. The three target articles are indispensable contributions in this regard. The admirable depth and scholarship demonstrated here will surely be an important collective asset for all of us who aspire to understand the human mind as fundamentally biocultural and to study it as such. 


\section{Acknowledgement}

We thank Jiyoung Park for her comments on an earlier draft.

\section{References}

Ames, D. \& Fiske, S. (2010). Cultural neuroscience. Asian Journal of Social Psychology, 13, 72-82.

Chiao, J. \& Ambady, N. (2007). Cultural neuroscience: Parsing universality and diversity across levels of analysis. In: S. Kitayama \& D. Cohen, eds. Handbook of Cultural Psychology, pp. 237-254. New York: Guilford Press.

Chiao, J. Y. \& Blizinsky, K. D. (2010). Culture-gene coevolution of individualism-collectivism and the serotonin transporter gene. Proceedings of the Royal Society B: Biological Sciences (in press).

Cochran, G. \& Harpending, H. (2009). The 10,000 Year Explosion: How Civilization Accelerated Human Evolution. New York: Basic Books.

Cooper, J., Zanna, M. P. \& Taves, T. A. (1978). Arousal as a necessary condition for attitude change following induced compliance. Journal of Personality and Social Psychology, 36, 1101-1106.

Draganski, B., Gaser, C., Busch, V., Schuierer, G., Bogdahn, U. \& May, A. (2004). Changes in grey matter induced by training. Nature, 427, 311-312.

Dunbar, R. (2007). Evolution of the social brain. In: S. W. Gangestad \& J. A. Simpson, eds. The Evolution of Mind: Fundamental Questions and Controversies, pp. 280-286. New York: Guilford Press.

Festinger, L. (1957). A Theory of Cognitive Dissonance. Stanford, CA: Stanford University Press.

Gutchess, A. H., Welsh, R. C., Boduroglu, A. \& Park, D. C. (2006). Cultural differences in neural function associated with object processing. Cognitive, Affective and Behavioral Neuroscience, 6, 102-109.

Han, S. \& Northoff, G. (2008). Culture-sensitive neural substrates of human cognition: A transcultural neuroimaging approach. Nature Reviews Neuroscience, 9 (8), 646-654.

Hanakawa, T., Honda, M., Okada, T., Fukuyama, H. \& Shibasaki, H. (2003). Neural correlates underlying mental calculation in abacus experts: Functional magnetic resonance imaging study. Neuroimage, 19, 296-307.

Hawks, J., Wang, E. T., Cochran, G. M., Harpending, H. C. \& Moyzis, R. K. (2007). Recent acceleration of human adaptive evolution. Proceedings of the National Academy of Sciences of the United States of America, 104, 20753-20758.

Ian, C., Weaver, G., Meaney, M. J. \& Moshe, S. (2006). Maternal care effects on the hippocampal transcriptome and anxietymediated behaviors in the offspring that are reversible in adulthood. Proceedings of the National Academy of Sciences of the United States of America, 103, 3480-3485.

Ishii, K., Kobayashi, Y. \& Kitayama, S. (2010). Interdependence modulates the brain response to word-voice incongruity. Social Cognitive and Affective Neuroscience (in press).
Ishii, K., Reyes, J. A. \& Kitayama, S. (2003). Spontaneous attention to word content versus emotional tone: Differences among three cultures. Psychological Science, 14, 39-46.

Kitayama, S., Conway, L. G., Pietromonaco, P. \& Park, H. (2010). Ethos of independence across regions of the U.S.: The production-adoption model of cultural change. American Psychologist (in press).

Kitayama, S. \& Park, J. (2010). Cultural neuroscience of the self: Understanding the social grounding of the brain. Social Cognitive and Affective Neuroscience (in press).

Kitayama, S., Park, H., Servincer, A. T., Karasawa, M. \& Uskul, A. K. (2009). A cultural task analysis of implicit independence: Comparing North America, West Europe, and East Asia. Journal of Personality and Social Psychology, 97, 236255.

Kitayama, S., Snibbe, A. C., Markus, H. R. \& Suzuki, T. (2004). Is there any 'free' choice? Self and dissonance in two cultures. Psychological Science, 15, 527-533.

Kitayama, S. \& Uskul, A. (2010). Culture, mind, and the brain: Current evidence and future directions. Annual Review in Psychology (in press).

Maguire, E. A., Gadian, D. G., Johnsrude, I. S., et al. (2000). Navigation-related structural changes in the hippocampi of taxi drivers. Proceedings of the National Academy of Sciences of the United States of America, 97, 4398-4403.

Mok, A. \& Morris, M. W. (2009). Cultural chameleons and iconoclasts: Assimilation and reactance to cultural cues in biculturals' expressed personalities as a function of identity conflict. Journal of Experimental Social Psychology, 45 (4), 884889.

Muente, T. F., Nager, W., Beiss, T., Schroeder, C. \& Altenmueller, E. (2003). Specialization of the specialized: Electrophysiological investigations in professional musicians. In: G. Avanzini, C. Faienza, D. Minciacchi, L. Lopez \& M. Majno, eds. The Neurosciences and Music, pp. 131-139. New York: New York Academy of Sciences.

Ng, S. H., Han, S., Mao, L. \& Lai, J. C. L. (2010). Dynamic bicultural brains: fMRI study of their flexible neural representation of self and significant others in response to culture primes. Asian Journal of Social Psychology, 13, 83-91.

Ross, L. \& Nisbett, R. E. (1991). The Person and the Situation: Perspectives of Social Psychology. New York: McGraw-Hill.

Schirmer, A. \& Kotz, S. A. (2003). ERP evidence for a sexspecific Stroop effect in emotional speech. Journal of Cognitive Neuroscience, 15 (8), 1135-1148.

Schwartz, S. H. (1992). Universals in the structure and content of values. In: M. Zanna, ed. Advances in Experimental Social Psychology, pp. 1-65. Orlando, FL: Academic Press.

Shweder, R. A. (1990). Cultural psychology: What is it? In: J. W. Stigler, R. A. Shweder \& G. Herdt, eds. Cultural Psychology: Essays on Comparative Human Development, pp. 1-46. Cambridge: Cambridge University Press.

Stiles, J. (2008). The Fundamentals of Brain Development: Integrating Nature and Nurture. Cambridge, MA: Harvard University Press.

van Praag, H., Kempermann, G. \& Gage, F. H. (1999). Running increases cell proliferation and neurogenesis in the adult mouse dentate gyrus. Nature Neuroscience, 2 (3), 266-270. 
van Veen, V., Krug, M. K., Schooler, J. W. \& Carter, C. S. (2009). Neural activity predicts attitude change in cognitive dissonance. Nature Neuroscience, 12 (11), 1469-1474.

Vul, E., Harris, C., Winkielman, P. \& Pashler, H. (2009). Puzzlingly high correlations in fMRI studies of emotion, personality, and social cognition. Perspectives in Psychological Scince, 4, 274-290.
Zhou, H. \& Cacioppo, J. (2010). Culture and the brain: Opportunities and obstacles. Asian Journal of Social Psychology, 13, 59-71.

Zhu, Y., Zhang, L., Fan, J. \& Han, S. (2007). Neural basis of cultural influence on self representation. Neuroimage, 34, 1310-1317. 